\title{
DGLAP versus perturbative Pomeron in hard diffractive processes large momentum transfer at HERA and LHC
}

\section{B.Blok ${ }^{* \dagger}$}

Physics Department Technion Haifa 32000 Israel

E-mail: blok@physics.technion.ac.il

\begin{abstract}
We study the energy dependence of the cross-section of the hard exclusive diffraction processes in QCD with large momentum transfer $-t$ in DGLAP approximation. We show that the results fully explain recent experimental data at HERA. Moreover, such processes at LHC energies and at $-t \geq M_{V}^{2}$ can be used as a "golden plate" ones to uncover the existence of perturbative pomeron, not masked by double logarithms.
\end{abstract}

XVIII International Workshop on Deep-Inelastic Scattering and Related Subjects, DIS 2010 April 19-23, 2010

Firenze, Italy

\footnotetext{
* Speaker.

${ }^{\dagger}$ The talk is based on recent work with L. Frankfurt and M. Strikman 1, 2]
} 


\section{Introduction.}

In this talk (see ref. [1, 2] for detailed discussion) we consider the hard inelastic diffractive (HID) processes with large momentum transfer $-t$ and large rapidity gap, like $\gamma+p \rightarrow J / \psi+$ rapidity gap $+X$ (Fig.1), that were studied at HERA recently [3, 4, 5]. We show that the specific model independent properties of the DGLAP approximation which are absent in the pQCD calculations of Pomeron exchange at large $-t$ ( cf. refs. [6, 7]) allow to describe the HERA data. In the DGLAP approximation the amplitudes are rapidly increasing with the incident energy since the $\log \left(x_{0} / x\right)$ terms that define the energy dependence of the amplitudes, are multiplied by large logarithms that arise from the integration over parton transverse momenta, leading to double log (DLA) asymptotics of the cross-section. Consider now the diffraction processes with large momentum transfer defined above. It was understood recently [2] that the cross section for diffractive charmonium photoproduction off a parton does not increase with energy for $-t \geq M_{J / \psi}^{2}$ in striking contrast with a rapid increase of the exclusive charmonium photoproduction at $t=0$ since logs arising from the integration over parton transverse momenta are $\log \left(M_{V}^{2} /\left(Q_{0}^{2}-t\right)\right)$, and thus disappear at $-t \sim M_{V}^{2}$. This result is valid in all orders of DGLAP approximation and thus the cross section of diffractive charmonium production off a parton is energy independent at large $-t$.

The dominance of the double logarithmic terms in a wide kinematic region in the two scale processes, shows that the multiRegge dynamics could be revealed only in the very special one scale processes where the $Q^{2}$ evolution is suppressed. The large $-t$ ultraperipheral processes at LHC represent an example of such phenomena. In the kinematical range $-t \geq M_{J / \psi}^{2}$ the double logarithmic (DL) terms are absent. So the HID phenomena represent a golden plated process for uncovering the onset of the $\mathrm{pQCD}$ Pomeron. Switching from HERA to ultraperipheral processes at LHC significantly increases the kinematical window allowed for the multiRegge kinematics. In this case there are up to $9 \div 10$ units in rapidity available for the multiRegge gluons. An unambiguous signature of these gluons will be a rapid increase of the diffractive cross section with energy in the region where DGLAP predicts the energy independent cross section.

\section{DGLAP description of HID processes: theory versus experiment.}

The quantative pQCD description of HID processes was recently developed in ref. [2]]. The differential cross section in the kinematic range $-t \leq Q^{2}+M_{V}^{2}$ is given by:

$$
\begin{gathered}
\frac{d \sigma}{d t d x_{J}}=\Phi\left(t, Q^{2}, M_{V}^{2}\right)^{2} \frac{\left(4 N_{c}^{2} I_{1}(u)\right)^{2}}{\pi u^{2}} G\left(x_{J}, t\right) . \\
u=\sqrt{16 N_{c} \log \left(x / x_{J}\right) \chi^{\prime}}, \chi^{\prime}=\frac{1}{b} \log \left(\frac{\log \left(\left(Q^{2}+M_{V}^{2}\right) / \Lambda^{2}\right)}{\log \left(-t+Q_{0}^{2}\right) / \Lambda^{2}}\right), \\
x_{J}=-t /\left(M_{X}^{2}-m_{p}^{2}-t\right), x \sim 3\left(Q^{2}+M_{V}^{2}\right) /(2 s), b=11-2 / 3 N_{f}, N_{c}=3, s=W_{\gamma p}^{2}
\end{gathered}
$$

The factor $\Phi\left(t, Q^{2}, M_{V}^{2}\right)$ in eq.2.1 is the energy independent function. The second factor corresponds to the distribution of gluons in a parton, calculated in the DL approximation, The last factor in eq. 2.1 is the gluon structure function of the nucleon that can be calculated using e.g. CTEQ6 
data (we neglect small contribution of the quark sea). In this equation $M_{X}^{2}$ is the invariant mass of the hadronic system produced due to the diffractive dissociation of a proton (see Fig. 1), $\Lambda=300$ $\mathrm{MeV}$, and $-t$ is the transverse momentum transfer. For the photoproduction processes $Q^{2}=0$. Let us stress that a characteristic feature of eq. 2.1 is the absence of the energy dependence at $-t \geq M_{V}^{2}$ (for $Q^{2}=0$ ).

Let us compare now the theoretical prediction with the recent experimental data. This comparison, as it was first noted in ref. [8], is not straightforward, since the HERA experiments, see e.g. [5], report the integral over invariant masses:

$$
\begin{gathered}
\frac{d \sigma(s, t)}{d t}=\int_{B(s)}^{A(s)} \frac{d M_{X}^{2}}{\left(M_{X}^{2}-t\right)^{2}} \frac{d^{2} \sigma}{d t d x_{J}}\left(x_{J}, s, t\right) \\
A(s)=0.05 s-t, B(s)=1 \mathrm{GeV}^{2},
\end{gathered}
$$

In order to compare the theoretical prediction with the experimental data we calculated the integral 2.3 numerically for all $-t$. We present the main results of our calculations in Fig. \&. We calculate the logarithmic derivative

$$
I(s, t)=\frac{1}{2} \frac{d \log (d \sigma / d t)}{d \log (s)}
$$

for $s=2 \cdot 10^{4} \mathrm{GeV}^{2}$ (we denote this quantity as $\alpha_{\mathbb{P}}^{\text {eff }}(t)$ ), and compare it with the data for $I(s, t)$ presented in Fig. 9 in ref. [5]. It is referred to in ref. [5] as the "Pomeron" trajectory $\alpha_{\mathbb{P}}(t)-1$. In the calculation we use CTEQ6M and CTEQ6L gluon parton distribution functions (pdf) $G\left(x_{J}, t\right)$. For small $-t\left(-t \sim 2 \mathrm{GeV}^{2}\right)$ the curves for such "effective Pomeron" are given by the dashed lines, since for these $-t$ the integration region includes the range of $x / x_{J} \sim 0.1-1$, where nondiagonal (GPD) effects not included in our treatment may be important. In addition, the gluon pdf's for moderate $Q^{2}$ are subject to significant uncertainties. The results are clearly within the experimental errors. For comparison we also depict in Fig. 2 the logarithmic derivative of the double differential cross section, given by eq. 2.1. which corresponds to the "true" Pomeron in the triple Pomeron limit.

Existing calculations of cross sections of large $t$ diffractive processes within perturbative Pomeron hypothesis, cf 价 predict qualitatively different interplay of $t$ and $W$ dependence compared to DGLAP. They predict that dependence on energy of the gluon pdf of the proton should be independent of $Q^{2}$ and perturbative Pomeron trajectory only weakly depends on $-t$, decreasing like $\alpha_{s}\left(M_{V}^{2}-t\right)$, in variance with the HERA data [5].

Let us stress the crucial role of the increase of the rate of energy dependence of $G\left(x_{J}, t\right)$ with the increase of $-t$, that is crucial for the explanation for the HERA data, in particular the increase of $\alpha_{\mathbb{I P}}$ with $-t$. This property is esily explained by DGLAP, but is absent in BFKL.

Observation that the pQCD Pomeron regime does not set in for the HERA kinematics can be understood as the consequence of the constraints due to the energy-momentum conservation (see refs. [1], 2] for references and more detailed discussion). There must be at least $2 \div 2.5$ units in rapidity for each gluon radiation in the multiRegge kinematics. This means that for the radiation of even one such gluon a rapidity window of at least $4 \div 5$ units in rapidity is required. One should 
add to this interval the 4-5 units of rapidity due to impact-factor and proton fragmentation. Thus it seems that there is no room in the HERA kinematics for the gluon radiation in the multiRegge kinematics.

\section{MultiRegge gluons in HID processes with large momentum transfer $-t$ at LHC.}

Consider now the ultraperipheral processes at the LHC (for a review see [9]). In this case one may have up to 14 units in rapidity, i.e. up to 9 units in rapidity may be available for a ladder describing gluon-parton scattering. A simple estimate suggests that for small $-t$ most of the kinematic range (even at the LHC) will be dominated by double However, as it is clear from the previous subsection, for $-t \geq M_{V}^{2}$ the double logarithmic terms are absent, and the entire increase of the double differential cross section $d^{2} \sigma /\left(d t d x_{J}\right)$ will be due to multi Regge gluons. In Fig. 3 we show(for illustrative purposes only ), the behavior of the $d^{2} \sigma /\left(d t d x_{J}\right)$ as a function of energy using currently popular pQCD Pomeron models with intercept $\alpha_{\mathbb{P}}^{\mathrm{BFKL} / \text { resummed }}(t)-1 \sim 0.2$.

\section{Conclusion}

We have shown that DGLAP predictions are in a good agreement with the behavior of HID processes observed at HERA. We found that the ultraperipheral collisions at LHC are a unique place where the onset of gluon radiation in the multiRegge kinematics may be observed in the near future.

\section{References}

[1] B. Blok, L. Frankfurt, M. Strikman, arXiv:1002.3048[hep-ph] , Phys. Lett. B, in press

[2] B. Blok, L. Frankfurt, M. Strikman,Eur.Phys.J.C67:99-109,2010.

[3] S. Chekanov et al. [ZEUS Collaboration], Eur. Phys. J. C 26, 389 (2003).

[4] A. Aktas et al [H1 Collaboration] Phys. Lett. B 568, 205 (2003); ibid B 638, 422 (2006).

[5] S. Chekanov et al. [ZEUS Collaboration], arXiv:0910.1235 [hep-ex].

[6] L. N. Lipatov, Sov. Phys. JETP 63 (1986) 904.

[7] R. Enberg, J. R. Forshaw, L. Motyka and G. Poludniowski, JHEP 0309, 008 (2003), ibid 0312, 002 (2003).

[8] L. Frankfurt, M. Strikman and M. Zhalov, Phys. Lett. B 670 (2008) 32.

[9] K. Hencken et al. Phys. Rept. 458 (2008) 1. 


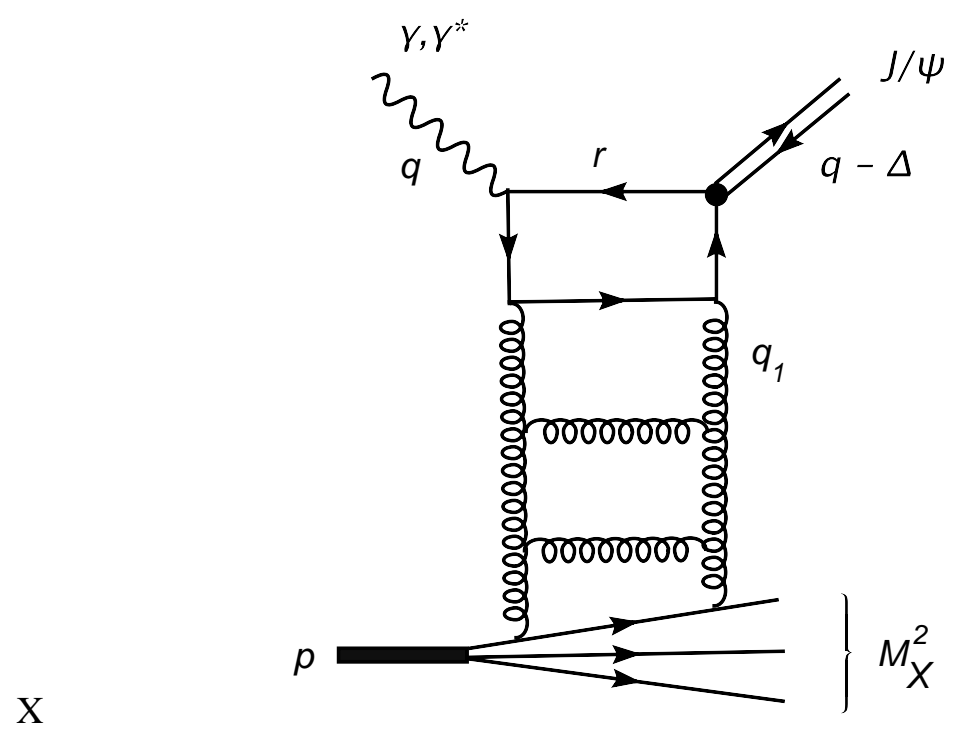

Figure 1: The Feynman diagram describing the double diffractive process in the triple "Pomeron" limit in pQCD (there is also a cross diagram, not depicted explicitly.

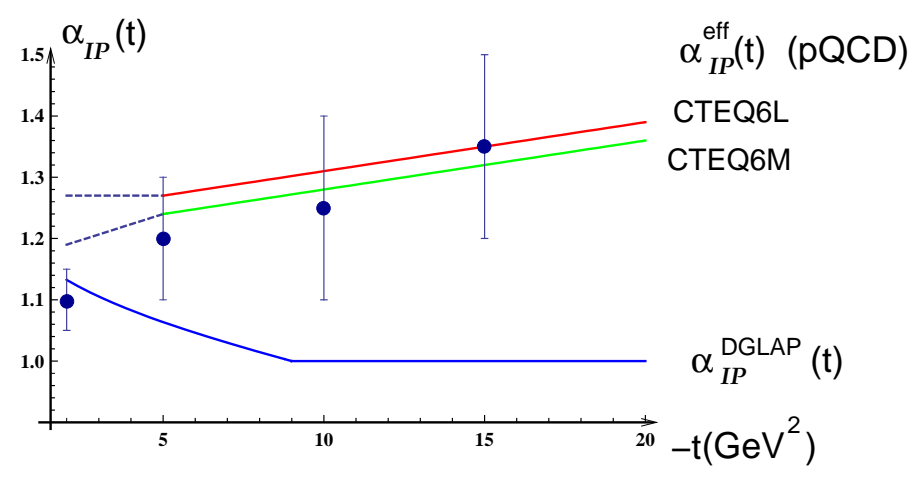

Figure 2: The comparison between the experimental data and theoretical prediction for the HID cross section at HERA for the "effective Pomeron" $\alpha_{\mathbb{P}}^{\text {eff }}(t)$, i.e. (1/2) logarithmic derivative of the cross section $d \sigma / d t$, obtained after integrating between the energy dependent cuts, as given in the text. The dashed curve means large theoretical uncertainties in the corresponding kinematic region. The values are given at for $W_{\gamma p}=150$ $\mathrm{GeV}$. In the same figure we depict also "true (DGLAP) "Pomeron", i.e. logarithmic derivative $\alpha_{\mathbb{P}}(t)^{\mathrm{DGLAP}}=$ $\frac{1}{2} \frac{d\left(d^{2} \sigma /\left(d t d x_{J}\right)\right)}{d \log \left(x / x_{J}\right)}$ at this energy. $\Lambda_{\mathrm{QCD}}=300 \mathrm{MeV}$. 


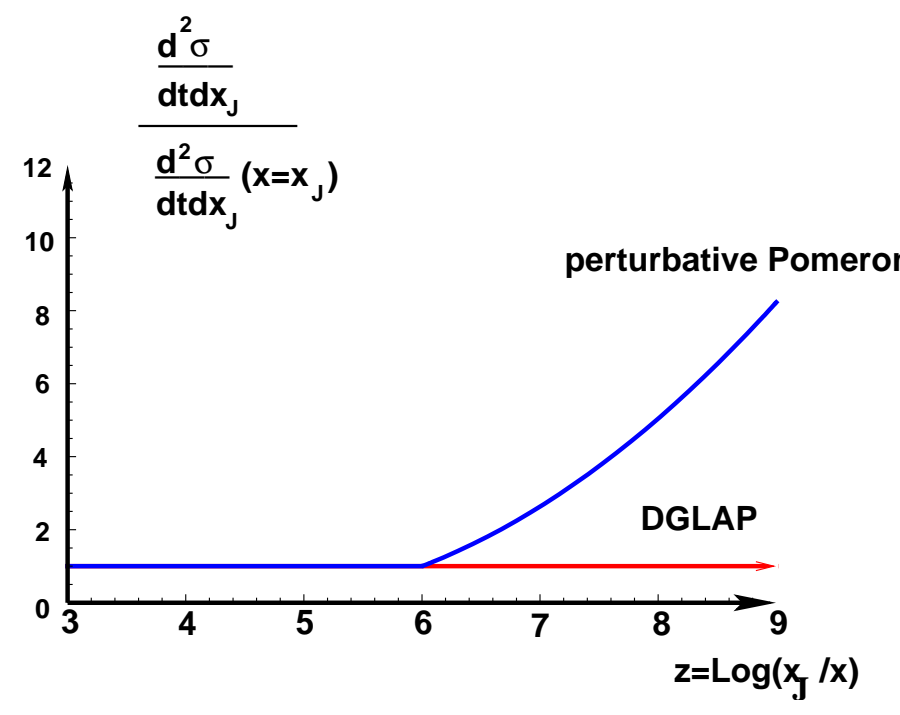

Figure 3: The increase of the cross section $d \sigma /\left(d t d x_{J}\right)$ with energy $\left(z=\log \left(x / x_{J}\right)\right)$ at LHC in DGLAP and perturbative "Pomeron" scenarios (for fixed $x_{J}$ ) 